\title{
Laparoscopic approach of cornual ectopic pregnancy after salpingectomy case report
}

Keywords: ectopic, pregnancy, potential risks, laparotomy, laparoscopic, excessive intraoperative, bleeding

\section{Introduction}

Cornual ectopic pregnancy (CEP) is a rare form of ectopic pregnancy with potential risks. Many of these cases are still converted to laparotomy nowadays, because of concerns about technical difficulties and risks of excessive intraoperative bleeding. We present an unusual case of an CEP in a patient diagnosed with infertility and pregnancy conceived by in vitro fertilization, which was managed by a laparoscopic approach.

\section{Case report}

A 40-year-old patient, nulliparous, with a history of adnexectomy in 2 occasions in the youth due to torsion of ovary due to benign tumor pathology on both occasions. It is performed the fifth of July of 2017 an in vitro fertilization treatment. Concurred on 30 of July of 2017 with pelvic pain and spotting. Laboratory at admission: hematocrit 41\%, Hemoglobin $14 \mathrm{~g} / \mathrm{dl}$, white blood cells $8500 / \mathrm{mm} 3$. Beta-HCG: 3100. A vaginal ultrasound is made that informs: endometrium of 8 $\mathrm{mm}$. In the left cornual area, a gestational sac containing a $2.3 \mathrm{~mm}$ embryo with positive cardiac activity (Figure 1).

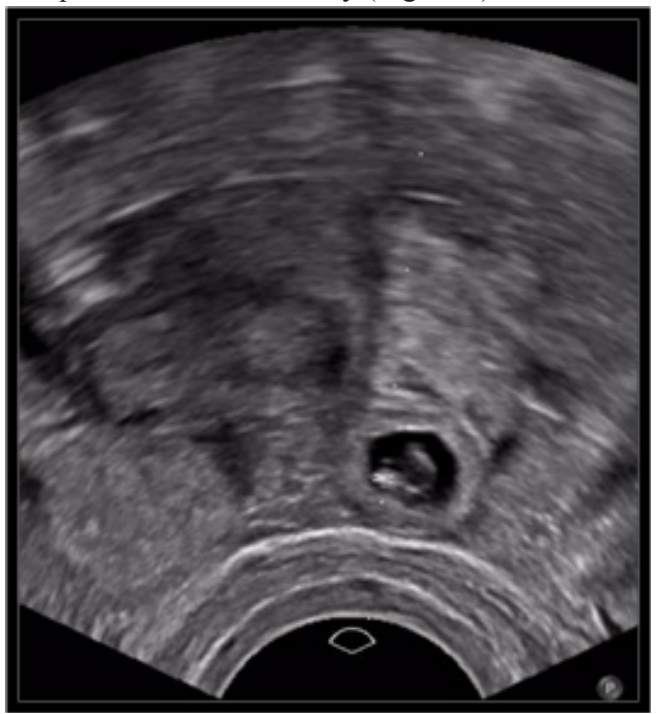

Figure I A gestational sac containing a $2.3 \mathrm{~mm}$ embryo with positive cardiac activity.

Laparoscopic approach was performed. A cornual resection was performed from the implantation base with meticulous hemostasia with monopolar electrosurgical energy (Hook). This caused minimal hemorrhage, without intraoperative complications. Subsequently, security coagulation in the surgical wound was performed with bipolar energy. The patient evolved favorably, being discharged at 12 hours after the procedures, without additional treatment requirements (Figure $2 \& 3$ ).
Volume 8 Issue 2 - 2019

\author{
R Illia,' Guallan F, Castaño Re, ${ }^{3}$ Habich D, ${ }^{4}$ \\ Ramilo Tp, ${ }^{5}$ Valencia Jaramillo V, ${ }^{6}$ Ratto $M c^{6}$ \\ 'Chief of Obstetrics Service, Department of Obstetrics and \\ Gynecology, Hospital Alemán, Argentina \\ ${ }^{2}$ Chief Resident, Department of Obstetrics and Gynecology, \\ Hospital Alemán, Argentina \\ ${ }^{3}$ Chief, Department of Obstetrics and Gynecology, Hospital \\ Alemán, Argentina \\ ${ }^{4}$ Chief of Gynecology Service, Department of Obstetrics and \\ Gynecology, Hospital Alemán, Argentina \\ ${ }^{5}$ Gynecological staff member, Gynecology Service, Department \\ of Obstetrics and Gynecology, Hospital Alemán, Argentina \\ ${ }^{6}$ Resident, Department of Obstetrics and Gynecology, Hospita \\ Alemán, Argentina
}

Correspondence: Ricardo Illia, Chief of Obstetrics Service, Department of Obstetrics \& Gynecology, Alemán Hospital, La Pampa 2219 5A, Argentina, Tel II61859985, Email rhillia@gmail.com

Received: August 19, 2018| Published: April 04, 2019

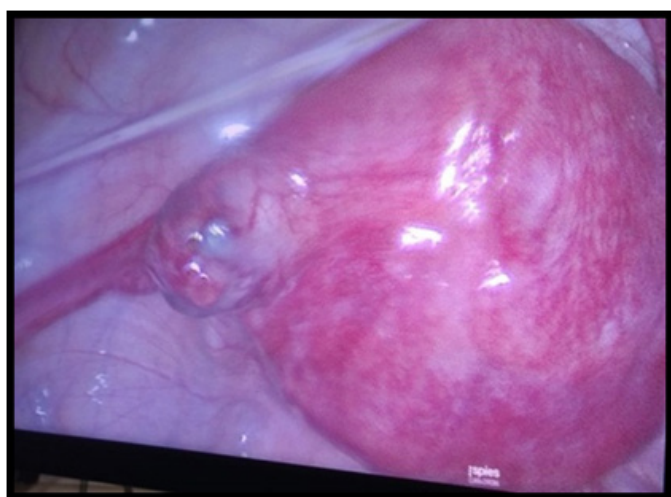

Figure 2 The patient evolved favorably, being discharged at 12 hours after the procedures, without additional treatment requirements.

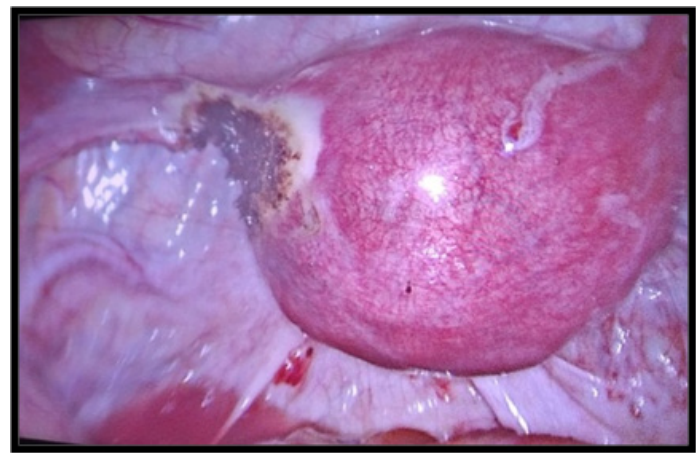

Figure 3 A security coagulation in the surgical wound was performed with bipolar energy.

\section{Discussion}

CEP were traditionally treated with laparotomy and wedge resection of the uterine horn or even hysterectomy. With the 
advancement of minimally invasive surgery, the laparoscopic approach offers a valuable option. At present there is no consensus as to which treatment is most advisable. We can divide them into surgical or non-surgical. Among the first have been described: cornuostomy, salpingostomy, Endoloop, myometrial incision with aspiration of the trophoblast and evacuation guided by hysteroscopy. Among the medical treatments the most widespread is the into the gestational sac injection of methotrexate or sodium chloride.

\section{Conclusion}

Cornual pregnancy is a very rare location, with high maternal morbidity and mortality, so an early diagnosis should be made to avoid complications and, if possible, a minimally invasive approach if feasible.

\section{Acknowledgments}

None.

\section{Conflicts of interest}

The author declares there are no conflicts of interest. 